\title{
Planet migration and magnetic torques
}

\author{
A. Strugarek ${ }^{1,2}$, A. S. Brun ${ }^{2}$, S. P. Matt ${ }^{3}$ and V. Reville ${ }^{2}$ \\ ${ }^{1}$ Université de Montréal, C.P. 6128 Succ. Centre-Ville, Montréal, QC H3C-3J7, Canada \\ email: strugarek@astro. umontreal.ca \\ ${ }^{2}$ CEA-Saclay, IRFU/SAp, Gif-sur-Yvette, France. \\ ${ }^{3}$ Department of Physics \& Astronomy, University of Exter, EX2 4QL, UK
}

\begin{abstract}
The possibility that magnetic torques may participate in close-in planet migration has recently been postulated. We develop three dimensional global models of magnetic starplanet interaction under the ideal magnetohydrodynamic (MHD) approximation to explore the impact of magnetic topology on the development of magnetic torques. We conduct twin numerical experiments in which only the magnetic topology of the interaction is altered. We find that magnetic torques can vary by roughly an order of magnitude when varying the magnetic topology from an aligned case to an anti-aligned case. Provided that the stellar magnetic field is strong enough, we find that magnetic migration time scales can be as fast as $\sim 100 \mathrm{Myr}$. Hence, our model supports the idea that magnetic torques may participate in planet migration for some close-in star-planet systems.
\end{abstract}

\section{Introduction}

The diversity of masses, sizes and orbits of known exoplanets has prompted recent efforts in the scientific community to explore the broad range of interactions that can exist between planets and their host stars (see Cuntz et al. 2000). In addition to tidal interactions (e.g. Mathis et al. 2013), planets orbiting inside the stellar wind Alfvén radius can magnetically interact with their host (Ip et al. 2004; Cohen et al. 2010; Strugarek et al. 2014). These interactions could lead to an angular momentum transfer between the planet and the star (Lovelace et al. 2008; Laine \& Lin 2011; Strugarek et al. 2014), resulting in a substantial planetary migration and participating in the dynamical (in)stability of the system. Among the star-planet interaction (SPI) models that have been developed, MHD simulations combine state of the art numerical models of cool stars magnetospheres and winds (Matt et al. 2012; Réville et al. 2015) with simplified models of planets (e.g., Cohen et al. 2014; Strugarek et al. 2014, and references therein). These global, dynamical models enable us to assess the effects of SPI in a self-consistent manner, by taking into account the full interaction channel from the planetary magnetosphere down to the lower stellar corona.

In a recent paper (Strugarek et al. 2015), we have developed MHD simulations of magnetic star-planet interactions in three dimensions. We address here the important question of the physical origin of the magnetic torque, and in particular the key role magnetic topology plays in determining the strength of the torque. We first briefly describe our methodology in Section 2, and detail how the magnetic torques are sustained in Section 3. We conclude in Section 4 by showing that for sufficiently strong stellar wind magnetic fields, the migration time-scale associated with magnetic torque is comparable with typical tidal migration time-scales. 


\section{Three-dimensional models of star-planet magnetic interaction}

We model magnetic star-planet interactions with global numerical simulation using the ideal MHD approach. We use the modular code PLUTO (Mignone et al. 2007) to solve the MHD equations with a standard HLL Riemann solver coupled to a secondorder Runge-Kutta method for the time integration. The soleinoidality of the magnetic field is enforced with a constrained method transport (see Strugarek et al. 2015). We discretize space with a cartesian grid in which a star and a planet are added as internal boundary conditions. At the stellar surface a magnetized stellar wind is imposed. We enforce a given magnetic field at the planetary boundary. We solve the MHD equations in the orbital rotating frame, such that the planet is fixed in the simulation grid. The star is positioned at the center of the simulation grid, and is hence as well fixed in the grid (the stellar rotation rate is corrected to account for the orbital rotating frame). The simulations are carried on a $490 \times 355 \times 355$ grid, with an resolution of $0.03 R_{\star}$ at the stellar boundary and a resolution of $0.06 R_{P}$ in the vicinity of the planet.

The modelled star is considered to be a typical cool star with a coronal temperature of $10^{6} \mathrm{~K}$, with a relatively strong magnetic field such that the Alfvén speed $v_{A}$ at the base of the corona is equal to the escape velocity $v_{\text {esc }}$ (for details see Strugarek et al. 2015). The star is considered to slowly rotate $\left(v_{\text {rot }}=3.0310^{-3} v_{\text {esc }}\right)$ and its wind is characterized by a large average Alfvén radius $\left\langle R_{A}\right\rangle=\sqrt{\dot{J} / \Omega_{\star} \dot{M}} \sim 18$ (see, e.g., Matt et al. 2012).

We consider a close-in planet located at $R_{\text {orb }}=5 R_{\star}$. The orbit is supposed to be circular since such close-in planet is likely to be tidally locked. We further assume that the planet possess an intrinsic dipolar magnetic field sufficiently large to retain a magnetosphere. The planetary magnetic field is chosen to be 10 times larger than the wind magnetic field at the planetary surface. We neglect any kind of planetary outflows (see Matsakos et al. 2015, for a complete discussion about such outflows) to focus on the effect of magnetic topology on the star-planet interaction itself. We consider the two cases of aligned and anti-aligned dipolar planetary fields.

We illustrate those two configurations in Figure 1 where a three-dimensional representation of the interaction is shown. In the aligned case (left panel), the magnetic topology allows the polar magnetic field lines of the planet (grey lines) to reconnect with the wind magnetic field lines (coloured lines). In the anti-aligned case (right panel), the planetary magnetosphere remains closed due to the incompatible topology of the two magnetic fields. We recall that only the orientation of the planetary field has been changed between the two cases, leaving all the other parameters untouched.

\section{Magnetic torques and planet migration}

The torques that develop in close-in star-planet systems can be separated into different physical contributions through (see Strugarek et al. 2015)

$$
\mathcal{T}=\mathcal{T}_{\text {ram }}+\mathcal{T}_{\text {Coriolis }}+\mathcal{T}_{\text {Magnetic tension }}+\mathcal{T}_{P}+\mathcal{T}_{\text {Magnetic pressure }}
$$

In Figure 2 we display these various contributions on the spherical cut symbolized by the white transparent sphere in Figure 1. The torques can be positive (red) or negative (blue), which respectively correspond to a loss or a gain of angular momentum for the orbiting planet, i.e. an infall or an expulsion of the planet. The torques are shown in Mollweide projection, with the central black circle delimiting the day-night meridian. As a result, the rightmost part of the projection corresponds to the upstream side of the interaction, and the leftmost side to the downstream side. In the aligned case the total 

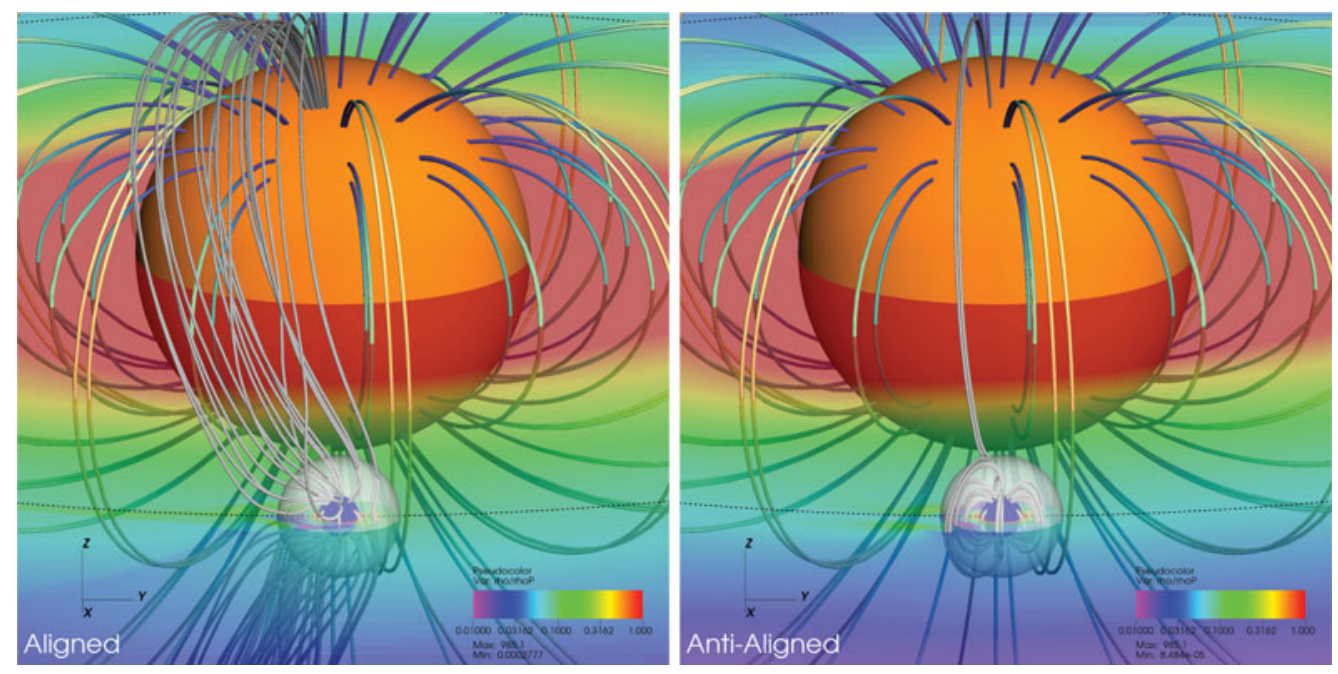

Figure 1. 3D renderings of the two star-planet interaction simulations. The stellar wind magnetic field lines are color-coded with the magnetic field strength, and the magnetic field lines connected to the planet are shown in grey. The stellar surface is represented by the orange sphere, and the planetary surface by the blue sphere. On the ecliptic the orbit is labeled by the black dashed line, and the logarithm of the density is shown by the transparent colormap. The transparent white sphere around the planet labels the integration sphere on which the torque components are calculated in Figure 2.

torque (bottom right panel) is completely dominated by magnetic tension (upper right panel) and magnetic pressure (bottom middle panel). Conversely, magnetic tension play almost no role in the anti-aligned case and the total torque is completely dominated by the magnetic pressure of the ambient wind magnetic field.

The magnetic pressure contribution is composed of positive and negative torques up and downstream, as expected from a stream-obstacle type of interaction. In the antialigned case, the obstacle can hence be simply approximated by the almost-spherical magnetosphere of the planet. In the aligned case, the magnetic tension is dominated by a positive torque downstream. The tension originates from the polar magnetic field lines that can be identified in Figure 1 (grey lines). In addition to the magnetosphere of the planet itself, these field lines act as a second obstacle and thus participate to the angular momentum exchange between the planet and its environment. The overall torque is obtained by integrating the various contributions over the integration sphere. As a result, only a small torque remains in the anti-aligned case, while magnetic tension provides a much stronger torque in the aligned case. The key role of topology clearly appears: in the aligned case the effective obstacle is completely different from the obstacle in the anti-aligned case, which results in a much weaker torque in the later case.

The migration time-scale associated with those torques is given by

$$
t_{P}=\frac{2 J_{P}}{\mathcal{T}}
$$

where the planetary orbital angular momentum is $J_{P}=M_{P}\left(G M_{\star} R_{\text {orb }}\right)^{1 / 2}$. The migration time-scale depends directly on the density normalization chosen for the stellar wind (see Strugarek et al. 2015, for a complete discussion), which controls the mass-loss rate and the magnetic field strength of the wind in physical units. For a surface stellar magnetic field $B_{\star} \sim 10 \mathrm{G}$, the migration time-scale is of the order of $1.410^{7}$ Myr in the 

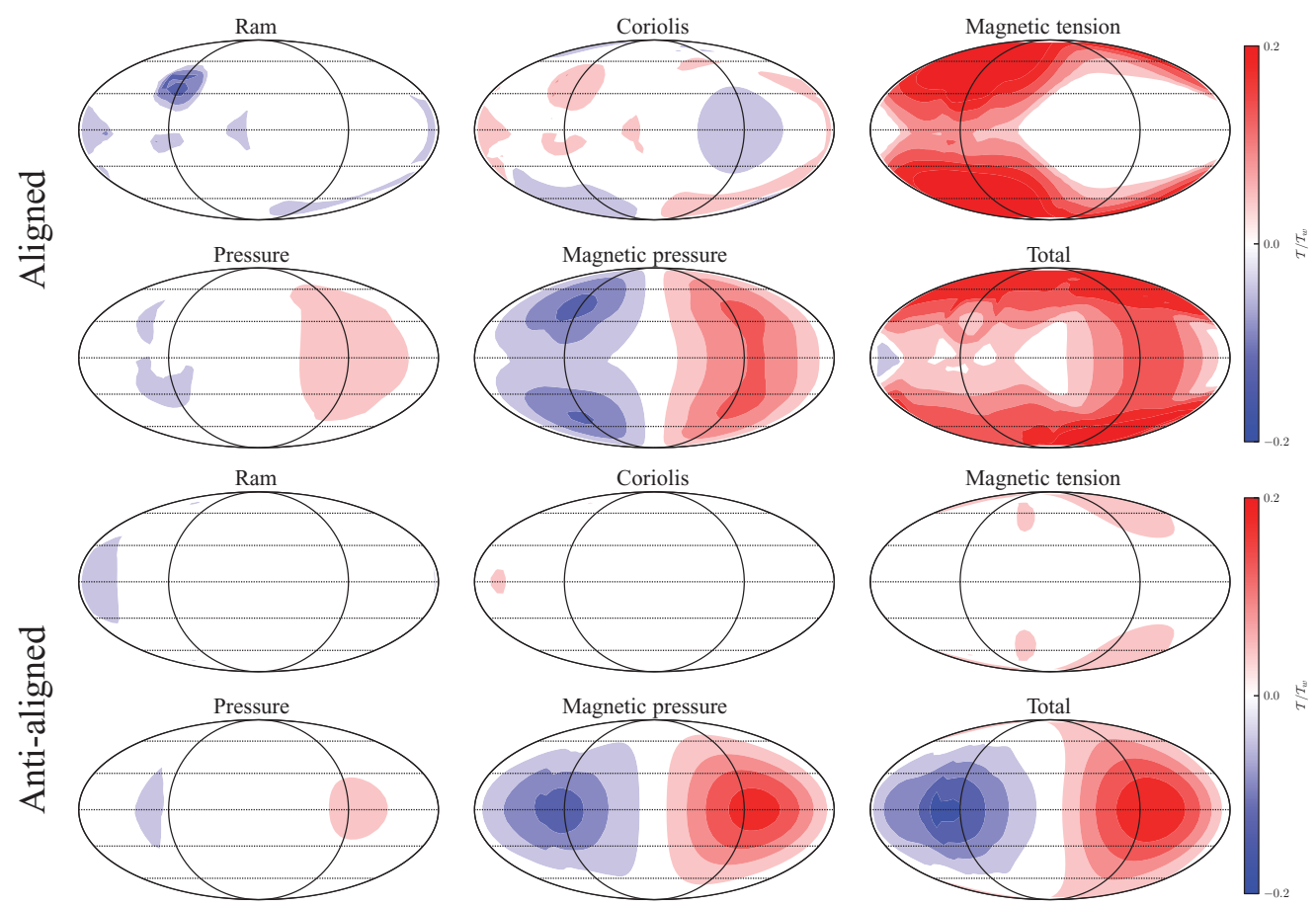

Figure 2. Contributions to the torque applied to the close-in planet. The terms are shown on the integration sphere of radius $0.3 R_{P}$ enclosing the planet with a Mollweide projection. The black circle in the center of each projection corresponds to the day-night delimitation. The upstream side is located on the right of each plot, and the downstream side on the left. The contributions are normalized by the stellar wind torque $\mathcal{T}_{w}$. Red denotes angular momentum extraction from the planet, while blue denotes deposition of angular momentum.

aligned case and $8.410^{7} \mathrm{Myr}$ in the anti-aligned case. As a result, for such moderate large-scale stellar magnetic fields, the magnetic torque does not induce any significant migration of the planet. On the contrary, for a surface stellar magnetic field $B_{\star} \sim 1 k G$, the migration time-scale drops by 4 orders of magnitude (hence a time-scale of $1.410^{3}$ Myr in the aligned case). In this case magnetic torques are able to compete with tidal torques for planet migration.

In conclusion, close-in planets can migrate due to magnetic torques provided the stellar magnetic field is sufficiently strong and the planet is sufficiently close. In addition, the magnetic topology has a key impact on the strength of the torque: by simply reversing the orientation of the planetary magnetic field, the torque is decreased by a factor of 6 .

\section{Conclusions}

We have demonstrated how magnetic topology changes close-in star-planet interactions using three-dimensional global numerical simulations. We developed twin simulations of close-in star-planet magnetic interactions in which we only changed the orientation of the planetary field. In the so-called aligned case, we showed that the effective planetary obstacle is much greater than the planetary magnetosphere alone. As a result, the magnetic torque is found to be 6 larger than in the conventional anti-aligned case. The magnetic torques are also shown to be sufficiently strong to compete with tidal torques for planet migration, provided the stellar magnetic field is sufficiently strong. These results confirm 
the first-order estimates that were derived in Strugarek et al. (2014) with simpler 2.5 numerical simulations. We are currently running a more extensive set of 3D models to empirically refine the torque scaling laws with orbital radius, magnetic field strengths and magnetic topology that were proposed in Strugarek et al. (2014).

Real stars posses much more complex magnetic fields than the simple dipolar configuration we considered here. In reality close-in planets are likely to interact with different local magnetic configurations along their orbit. Assessing what average magnetic torque results from a magnetic topology varying along the orbit will require dedicated $3 \mathrm{D}$ simulations tackling the dynamical aspects of a planet orbiting in a non-homogenous corona. Indeed, the time-scale on which the equilibrated configurations modelled in this work establish depends on the resistivity of the magnetospheric plasma of the planet, and on its reconnection efficiency with the stellar wind magnetic field. The numerical model presented in this work provides a solid basis for further, more realistic studies of star-planet magnetic interactions in which these dynamical aspects could be explored.

\section{References}

Cohen, O., Drake, J. J., Glocer, A., et al. 2014, ApJ, 790, 57

Cohen, O., Drake, J. J., Kashyap, V. L., Sokolov, I. V., \& Gombosi, T. I. 2010, ApJ, 723, L64

Cuntz, M., Saar, S. H., \& Musielak, Z. E. 2000, ApJ, 533, L151

Ip, W.-H., Kopp, A., \& Hu, J.-H. 2004, ApJ, 602, L53

Laine, R. O. \& Lin, D. N. C. 2011, ApJ, 745, 2

Lovelace, R. V. E., Romanova, M. M., \& Barnard, A. W. 2008, MNRAS, 389, 1233

Mathis, S., Alvan, L., \& Remus, F. 2013, EAS Publications Series, 62, 323

Matsakos, T., Uribe, A., \& Königl, A. 2015, A\&̛ A, 578, A6

Matt, S. P., MacGregor, K. B., Pinsonneault, M. H., \& Greene, T. P. 2012, ApJL, 754, L26

Mignone, A., Bodo, G., Massaglia, S., et al. 2007, ApJS, 170, 228

Réville, V., Brun, A. S., Matt, S. P., Strugarek, A., \& Pinto, R. F. 2015, ApJ, 798, 116

Strugarek, A., Brun, A. S., Matt, S. P., \& Réville, V. 2014, ApJ, 795, 86

—. 2015, Submitted to ApJ 